\title{
Anticonvulsant Hypersensitivity Syndrome
}

\section{In Vitro Assessment of Risk}

Neil H. Shear and Stephen P. Spielberg

With the technical assistance of Marilyn Cannon ${ }^{\dagger}$ and Margaret Miller

Divisions of Dermatology and Clinical Pharmacology, Departments of Pediatrics, Medicine, and Pharmacology, The Hospital for Sick Children; and Sunnybrook Medical Centre,

University of Toronto, Toronto, Ontario, Canada M5G 1 X8

\begin{abstract}
Arene oxide metabolites of aromatic anticonvulsants (phenytoin, phenobarbital, and carbamazepine) may be involved in the pathogenesis of hypersensitivity reactions. We investigated 53 patients with clinical sensitivity to anticonvulsants by exposing their lymphocytes in vitro to drug metabolites generated by a murine hepatic microsomal system. The diagnosis of a hypersensitivity reaction was corroborated by in vitro rechallenge for each drug (phenytoin, $n=34$; phenobarbital, $n=22$; carbamazepine, $n=25$ ) when cytotoxicity ( $\%$ dead cells) exceeded 3 SD above the mean result for controls. Cross-reactivity among the drugs was noted. 7 out of 10 patients who had received all three anticonvulsants had adverse reactions to each. 40 out of 50 patients tested to all three drugs in vitro were positive to each. Adverse reactions were indistinguishable among anticonvulsants. Skin rash (87\%), fever (94\%), hepatitis (51\%), and hematologic abnormalities (51\%) were common clinical features of each drug. $62 \%$ of reactions involved more than two organs. Cells from patients' parents exhibited in vitro toxicity that was intermediate between values for controls and patients. In vitro testing can help diagnose hypersensitivity to anticonvulsants. Cells from patients may also be used for prospective individualization of therapy to decrease risk of adverse reaction. Cross-reactivity among the major anticonvulsants is common and should be considered before deciding on alternative therapy.
\end{abstract}

\section{Introduction}

The anticonvulsant effect of phenytoin was described by Merritt and Putnam in 1938 (1). In their review of toxic symptoms of therapy, two cutaneous reactions were identified (2). The first was a mild, morbilliform eruption that cleared up when the drug was stopped and often did not recur when therapy was reinstituted. This was a common side-effect (5\% of patients), which now is believed to be dose related, but the exact pathogenesis remains unknown (3). The second was a severe, exfoliative dermatitis that was associated with fever and eosin-

Address reprint requests to Dr. N. Shear, Division of Clinical Pharmacology and Toxicology, The Hospital for Sick Children, 555 University Avenue, Toronto, Ontario, Canada M5G 1 X8.

Received for publication 15 January 1988 and in revised form 27 June 1988.

${ }^{\dagger}$ Deceased.

J. Clin. Invest.

(c) The American Society for Clinical Investigation, Inc.

$0021-9738 / 88 / 12 / 1826 / 07 \$ 2.00$

Volume 82, December 1988, 1826-1832 ophilia in $<1 \%$ of their patients. The systemic nature of this rarer complication was described 11 years later (4).

Unwelcome hypersensitivity reactions have been described for other established drugs (e.g., sulfonamides) and have been responsible for the demise of otherwise promising therapeutic agents (e.g., ticrynafen and zomepirac). This could not have been predicted by existing animal models, nor by the monitoring of serum drug concentrations.

Furthermore, such adverse events are rare and the possibility that a drug may cause hypersensitivity reactions can be missed during early human exposures. The low reaction rate suggests that a subpopulation may be at risk of developing idiosyncratic toxicity and that this might be secondary to a genetic defect of drug metabolism. We have undertaken the study of aromatic anticonvulsants as a model to help understand the pathogenesis and genetics of idiosyncratic reactions and to develop approaches to diagnosis and prediction.

Hypersensitivity reactions, as defined in this context, are characteristically rare (1:10,000 exposures), are delayed in onset after initiation of drug therapy, and can cause severe morbidity or result in death. The "phenytoin reaction" is described as a febrile illness associated with skin rash, lymphadenopathy, possible hepatitis, nephritis, pneumonitis, or hematologic abnormalities (5-11). In 1953 McGeachy and Bloomer described an identical syndrome secondary to phenobarbital (12). Their three patients had severe (two were fatal) reactions to therapeutic doses of phenobarbital characterized by fever, delirium, exfoliative dermatitis, hepatitis, and nephritis. Hepatitis secondary to phenobarbital is not discussed in standard reference texts but has been well described in at least nine cases (13). Carbamazepine hypersensitivity has also been reported with skin rash (including toxic epidermal necrolysis), fever, hepatitis, bone marrow toxicity, pneumonitis, and pseudolymphoma (14-18).

Problems with both diagnosis and prediction may arise when anticonvulsant toxicity occurs. Diagnostic confusion can occur with infection, collagen vascular disease, or lymphoma (19). Also, if a patient is being treated with more than one anticonvulsant it is difficult to differentiate which drug may be responsible for the reaction, and diagnostic rechallenge may result in severe reactions in the truly drug-sensitive patient. Potential cross-reactivity among the three major anticonvulsants limits the choices for future therapy in a patient who has had a reaction $(20,21)$.

Phenytoin, phenobarbital, and carbamazepine are metabolized to hydroxylated aromatic compounds. This would suggest that arene oxides are intermediates in the reaction, the result of oxidative (cytochrome P-450) metabolism (Fig. 1) $(22,23)$. Arene oxides generated in vivo are capable of binding to cellular macromolecules, thereby perturbing cell function and initiating immunologic responses (24). Our previous stud- 


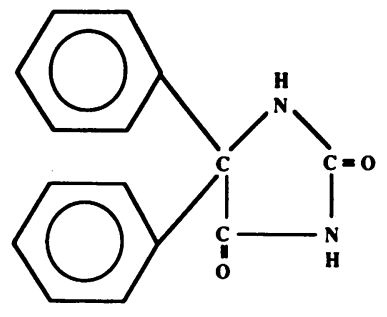

PHENYTOIN

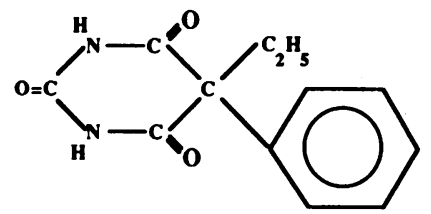

PHENOBARBITAL

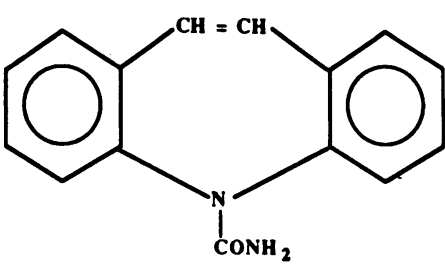

CARBAMAZEPINE

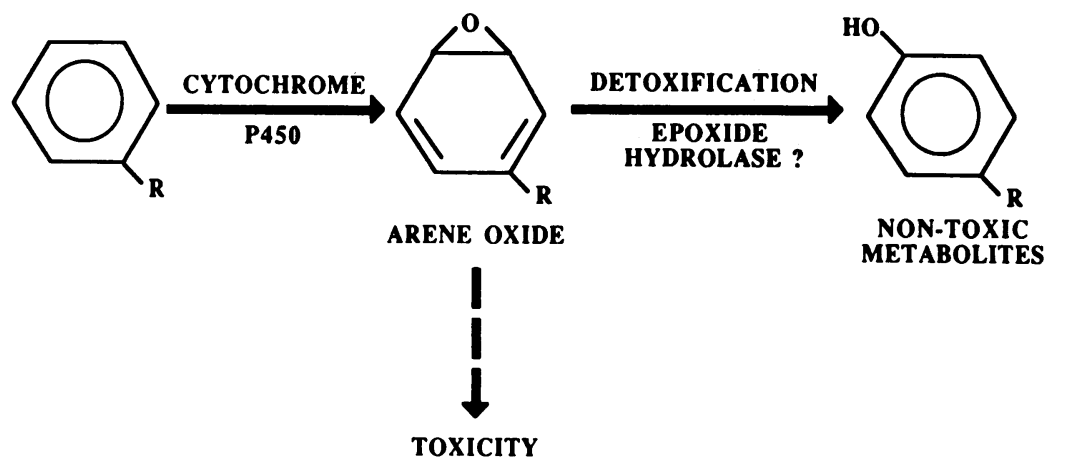

Figure 1. Anticonvulsant structures and the proposed metabolic pathway for arene oxide formation. The nontoxic metabolites include parahydroxylated compounds (as shown) as well as other products (e.g., hydroxylated, dihydroxylated, dihydrodiols, and diols). Detoxification is achieved in part spontaneously and in part by epoxide hydrolases. Toxicity is initiated by covalent binding of the reactive metabolites (arene oxides) to cellular macromolecules. The full mechanism of toxicity is unclear (22-24). ies have suggested that abnormal detoxification of arene oxides might predispose patients to toxicity from phenytoin and similar compounds $(11,25-27)$. A cellular model is necessary to investigate the pathogenesis of anticonvulsant hypersensitivity reactions and genetic defects in detoxification. The metabolic pathway is modeled in vitro by exposing peripheral blood lymphocytes to metabolites generated by a source of cytochrome P-450 (a mouse hepatic microsomal system). Lymphocytes are chosen, not as immunologic cells, but as easily obtained cells that contain epoxide hydrolases, enzymes involved in arene oxide detoxification. An in vitro cellular model allows safe rechallenge for the confirmation of drug sensitivity and the prediction of future toxicity risk of old and new anticonvulsants.

\section{Methods}

Subjects. Control subjects included 49 normal volunteers (aged 20-45 yr) never exposed to anticonvulsants and 10 patients (aged 20-35 yr) with seizure disorders who were chronically treated with phenytoin without adverse reactions.

Patients. 53 patients with suspected hypersensitivity reactions were studied by the in vitro lymphocyte toxicity assay, including three patients with phenytoin hepatotoxicity and one patient with anticonvulsant-induced aplastic anemia previously reported $(11,26)$. Patients were referred to us for diagnostic confirmation of a drug-induced reaction and/or to assist in the choice of future therapy. When possible, the parents and siblings of patients were studied.

In vitro lymphocyte toxicity assay. The in vitro assay for drug-metabolite-induced toxicity has been previously reported in detail $(25$, 28). Lymphocytes were prepared from whole blood with Ficoll-Paque and then suspended in a Hepes-buffered medium to yield $10^{6} \mathrm{cells} / \mathrm{re}-$ action. Hepatic microsomes were prepared from National Institutes of Health General Purpose Swiss Mice [N: GP(SW)] pretreated with phenobarbital $(10 \mathrm{mg} / \mathrm{kg}$ of body weight, given intraperitoneally for $3 \mathrm{~d}$ ). $0.28 \mathrm{mg}$ microsomal protein was incubated with the lymphocytes at $37^{\circ} \mathrm{C}$ for $2 \mathrm{~h}$ along with $0.6 \mathrm{mM}$ NADP, $2.4 \mathrm{mM}$ glucose-6-phosphate, $2 \mathrm{U}$ of glucose-6-phosphate dehydrogenase, and drug (phenytoin, phenobarbital, or carbamazepine). A single concentration $(62.5 \mu \mathrm{M})$ was chosen that approximated in vivo conditions and that was derived from dose-toxicity studies. The results for phenytoin have been published previously $(25,26)$ and results for phenobarbital and carbamazepine are similar (unpublished data). The drug was added in DMSO (final concentration, 2.5\%). The same concentration of DMSO was included in baseline samples lacking drug. Cells were collected by centrifugation and resuspended in Hepes-buffered medium containing $5 \mathrm{mg}$ of albumin $/ \mathrm{ml}$. Incubations were continued at $37^{\circ} \mathrm{C}$ for $16 \mathrm{~h}$ and aliquots then taken for lymphocyte viability assessment by trypan blue dye exclusion. At least 200 cells/sample were examined in a blind protocol.

These experiments were reviewed and approved by the Scientific and Ethics Review Committees of the Hospital for Sick Children. Informed consent was obtained from all individuals under study.

Statistical analysis. Each experimental condition (no drug, phenytoin, phenobarbital, and carbamazepine) was studied in triplicate samples and the mean percent of dead lymphocytes was determined. The coefficient of variation was $<10 \%$ for all experiments. The change from baseline values (calculated as percent dead cells in the presence of drug minus the percent dead cells in the absence of drug) was used as the measure of toxicity. Toxicity values from patients and relatives that were above the upper limit for normals $(\mu+3 \mathrm{SD})$ were considered positive. Differences among groups were determined by the use of confidence intervals, analysis of variance, and Duncan multiple range test.

\section{Results}

Clinical features of patients (Table I). The age range in the patients studied was from 1 to $76 \mathrm{yr} .33$ patients were younger than $18 \mathrm{yr}$ ( 20 male, 13 female) and 20 were adults ( 9 male, 11 female).

The clinical presentation of a typical reaction was with the onset of fever a mean of $21 \mathrm{~d}$ (range 7 to $90 \mathrm{~d}$ ) after beginning 


\begin{tabular}{|c|c|c|c|c|c|c|c|c|c|c|c|}
\hline Patient & Age & $\operatorname{Sex}^{*}$ & Drug & Skin & Liver & Kidney & Lung & Blood & Eos & $\begin{array}{c}\text { Atyp } \\
\text { lymph }\end{array}$ & Other \\
\hline & $y r$ & & & & & & & & & & \\
\hline 1 & 44 & $\mathbf{M}$ & DPH & EM & $x$ & & $x$ & & & & \\
\hline 2 & 16 & $\mathbf{M}$ & DPH & E & & & & & & & \\
\hline 3 & 4 & $\mathbf{M}$ & DPH & & & & & PMN & & & \\
\hline 4 & 7 & $\mathbf{M}$ & PB & E & & & & & & $x$ & \\
\hline 5 & 16 & $\mathbf{F}$ & DPH & E & $x$ & $x$ & & & & & \\
\hline 6 & 1 & $\mathbf{M}$ & PB & $\mathbf{E}$ & $x$ & & & & $x$ & $x$ & \\
\hline 7 & 13 & $\mathbf{F}$ & CARB & E & $x$ & $x$ & & $\mathrm{C}+\mathrm{HA}$ & & & Heart, brain, ANA \\
\hline 8 & 14 & $\mathbf{F}$ & DPH & E & & & & & & & \\
\hline 9 & 16 & $\mathbf{M}$ & CARB & E & $x$ & & & & $x$ & & \\
\hline 10 & 16 & $\mathbf{M}$ & DPH & E & $x$ & & & & $x$ & & \\
\hline 11 & 7 & $\mathbf{F}$ & DPH & E & $x$ & & & & $x$ & & \\
\hline 12 & 30 & $\mathbf{M}$ & CARB & E & & & & & & & \\
\hline 13 & 26 & $\mathbf{M}$ & DPH & E & $x$ & & $x$ & & & & \\
\hline 14 & 8 & $\mathbf{M}$ & DPH & E & & & & PMN & & & \\
\hline 15 & 14 & $\mathbf{M}$ & DPH & E & & & & & $x$ & & \\
\hline 16 & 18 & $\mathbf{F}$ & CARB & & $x$ & & & PMN & & & \\
\hline 17 & 11 & $\mathbf{M}$ & DPH & E & $x$ & & & & & & \\
\hline $18^{\ddagger}$ & 53 & $\mathbf{M}$ & CARB & & $x$ & & & APLAS & & & \\
\hline 19 & 5 & $\mathbf{M}$ & DPH & $\mathbf{E}$ & & & & & & & \\
\hline 20 & 7 & $\mathbf{M}$ & CARB & & & $x$ & & PLAT & & & \\
\hline 21 & 7 & $\mathbf{M}$ & CARB & $\mathbf{E}$ & & & & & & & \\
\hline 22 & 1 & $\mathbf{M}$ & CARB & & $x$ & & & & & & \\
\hline 23 & 11 & $\mathbf{F}$ & CARB & E & & & & & & & \\
\hline 24 & 30 & $\mathbf{F}$ & DPH & E & $x$ & & $x$ & & $x$ & & \\
\hline $25^{t}$ & 21 & F & DPH & E & $x$ & & & & $x$ & & ANA \\
\hline $26^{4}$ & 30 & F & DPH & E & $x$ & & & $\mathrm{C}+\mathrm{HA}$ & & & \\
\hline $27^{6}$ & 27 & $\mathbf{F}$ & DPH & E & $x$ & & & & $x$ & & \\
\hline 28 & 11 & $\mathbf{F}$ & DPH & $\mathrm{E}$ & $x$ & & & & $x$ & & \\
\hline 29 & 56 & $\mathbf{F}$ & DPH & E & & & & & & & \\
\hline 30 & 37 & $\mathbf{F}$ & CARB & E & & & & PMN & & & \\
\hline 31 & 6 & $\mathbf{M}$ & CARB & E & & & & & & & \\
\hline 32 & 76 & $\mathbf{F}$ & DPH & E & & & & & & & \\
\hline 33 & 16 & $\mathbf{F}$ & CARB & E & & & & & $x$ & & \\
\hline 34 & 19 & $\mathbf{F}$ & DPH & E & $x$ & $x$ & $x$ & PMN & $x$ & & Heart \\
\hline 35 & 2 & $\mathbf{F}$ & PB & E & $x$ & & $x$ & & $x$ & $x$ & Thyroid \\
\hline 36 & 10 & $\mathbf{F}$ & DPH & E & $x$ & & & & & & Brain \\
\hline 37 & 60 & $\mathbf{M}$ & DPH & TEN & & & & & & & \\
\hline 38 & 3 & $\mathbf{M}$ & PB & TEN & $x$ & & & PMN & $x$ & & \\
\hline 39 & 10 & $\mathbf{F}$ & CARB & $\mathrm{E}$ & & & & & & & \\
\hline 40 & 29 & $\mathbf{F}$ & DPH & & $x$ & & & PMN & & & \\
\hline 41 & 16 & $\mathbf{F}$ & DPH & E & & & & & & & \\
\hline 42 & 4 & $\mathbf{M}$ & CARB & TEN & & & & PMN & & & \\
\hline 43 & 50 & $\mathbf{M}$ & DPH & E & & & & & $x$ & & \\
\hline 44 & 75 & $\mathbf{M}$ & DPH & E & & $x$ & & & $x$ & & \\
\hline 45 & 4 & $\mathbf{M}$ & PB & EM & & & & & & & \\
\hline 46 & 4 & $\mathbf{M}$ & DPH & E & $x$ & & & & & & \\
\hline 47 & 14 & $\mathbf{M}$ & DPH & E & & & & & & & \\
\hline 48 & 30 & $\mathbf{F}$ & DPH & E & $x$ & & & & & & \\
\hline 49 & 49 & $\mathbf{M}$ & DPH & $\mathbf{E}$ & & $x$ & & & & & \\
\hline 50 & 4 & $\mathbf{F}$ & PB & E & $\times$ & & & & & & \\
\hline 51 & 9 & $\mathbf{M}$ & DPH & E & $x$ & & & & $x$ & & Thyroid \\
\hline 52 & 8 & $\mathbf{F}$ & PB & E & & & & & & & \\
\hline 53 & 23 & $\mathbf{M}$ & DPH & E & $x$ & & & & & & Pancreas \\
\hline Total & & & & 47 & 27 & 6 & 5 & & 16 & 3 & \\
\hline
\end{tabular}

Target organ involvement during the first reaction to an anticonvulsant. * Abbreviations in the table: M, male; F, female; DPH, phenytoin; PB, phenobarbital; CARB, carbamazepine; E, exanthematous; EM, erythema multiforme; TEN, toxic epidermal necrolysis; PMN, agranulocytosis; C+HA = Coombs' positive hemolytic anemia; APLAS, aplastic anemia; PLAT, thrombocytopenia; ANA, positive antinuclear antibodies; Eos, eosinophilia; Atyp lymph, atypical lymphocytosis. ${ }^{\ddagger}$ Previously reported (reference 11). $\$$ Previously reported (reference 27).

anticonvulsant therapy. This was followed by the development of a skin rash $(87 \%$ of patients) that varied in severity from a faint generalized exanthematous eruption to toxic epidermal necrolysis. Manifestations of internal organ involvement tended to be subsequent to this, most commonly hepatitis (51\%) and nephritis (11\%). Multi-organ involvement (i.e., two 
Table II. Results of the In Vitro Lymphocyte Toxicity Assay

\begin{tabular}{|c|c|c|c|c|c|c|}
\hline \multirow[b]{2}{*}{ Drug } & \multicolumn{3}{|c|}{ Controls } & \multicolumn{3}{|c|}{ Patients* } \\
\hline & $n$ & Mean & $\begin{array}{l}\text { Upper limit } \\
\text { of normal }\end{array}$ & $n$ & Mean & $\begin{array}{l}\text { 95\% Confidence } \\
\text { limits }^{8}\end{array}$ \\
\hline & \multicolumn{4}{|c|}{$\begin{array}{c}\% \text { dead cells above } \\
\text { baseline }\end{array}$} & \multicolumn{2}{|c|}{$\begin{array}{c}\% \text { dead cells above } \\
\text { baseline }\end{array}$} \\
\hline Phenytoin & 53 & 0.1 & 3.6 & 34 & 13.5 & $11.7-15.3$ \\
\hline Phenobarbital & 38 & 0.4 & 4.5 & 22 & 13.3 & $10.2-16.5$ \\
\hline Carbamazepine & 37 & 3.6 & 9.9 & 25 & 20.6 & $17.5-23.8$ \\
\hline
\end{tabular}

* Patients who had sustained clinical hypersensitivity reactions to the anticonvulsant.

${ }^{\ddagger}$ SD above the mean.

$\S$ Mean \pm 2.0 SEM.

or more major organs involved, not including eosinophilia, atypical lymphocytosis, and fever) was noted in 31 patients. Of the remaining 22 patients, 20 had skin rash alone. When present, atypical lymphocytosis occurred in the first week of the reaction and eosinophilia was noted by the second week. Arthralgia and arthritis were notably absent in our series. In some patients, despite discontinuation of anticonvulsant therapy at the onset of a reaction, manifestations of toxicity occurred in previously uninvolved organs, or increased in organs already involved.

In vitro toxicity assay. In the absence of microsomes or the NADPH-generating system phenytoin, phenobarbital, or carbamazepine are not toxic to cells from controls or patients.

Toxicity of the anticonvulsant metabolites to cells from controls is shown in Table II. There was no difference between normals and patients who could tolerate phenytoin. Toxicity of the metabolites to cells from patients with a clinical history suggestive of a hypersensitivity reaction differ significantly from controls $(P<0.001)$ (Table II).

Family studies. Toxicity of phenytoin-metabolites to cells from controls, patients who had sustained hypersensitivity reactions to phenytoin, and the parents of seven of those patients is shown in a histogram (Fig. 2). The three populations are statistically distinct $(P<0.001)$. The parent (intermediate) group has a mean of $5.6 \%$ dead cells above baseline $( \pm 0.3 \mathrm{SEM})$.

The results from two families are shown in Fig. 3. Two siblings in the first family (subjects 5 and 6) both received phenytoin therapy for seizure control, and the results of in vivo and in vitro exposure correlate. Subject 5 was able to continue phenytoin therapy while subject 6 could not because of a hypersensitivity reaction. In family 2 , two of the siblings were normal by in vitro testing and two were abnormal. Parents from both families had in vitro results that were intermediate to the control (normal) and patient populations.

Clinical-in vitro correlation. The broad range (Fig. 2) of results suggests that a quantitative relationship might exist between in vitro results and clinical toxicity. Rather, no relationship was apparent between cell toxicity and latency of reaction onset or degree of reaction (number or nature of organ involvement, severity, time for recovery).

The correlation of clinical (in vivo) exposure to an anticonvulsant with in vitro results is shown in Table III. 34 patients who received phenytoin therapy had adverse reactions (of the delayed hypersensitivity-multiorgan type) and all of these had positive in vitro test results (i.e., true positive). One patient (number 22) was treated chronically with phenytoin with no adverse reactions and had normal in vitro test results (true negative). No false positive or false negative results were noted.

The results for carbamazepine were similar, with 25 true positive and 2 true negative results.

For phenobarbital there were 21 true positive and 3 true negative results. However, 2 false positive results (patients 31 and 47) and 1 false negative result (patient 3 ) were obtained.

Clinically, 10 patients had been treated with all three anticonvulsants and seven (70\%) had hypersensitivity reactions with each course of therapy. Lymphocytes from six of these seven patients were positively sensitive to metabolites of all three of the anticonvulsants in vitro. The other patient's cells were sensitive to phenytoin and carbamazepine.

Lymphocytes from 50 patients were tested to all three anticonvulsants. 40 patients $(80 \%)$ were sensitive in vitro to all three drugs, 4 were sensitive to a single drug (phenytoin $=2$, carbamazepine $=2$ ), and 6 were sensitive to two drugs.

One patient (patient 18) had had clinical reactions to phenytoin and carbamazepine (11). In vitro testing suggested phenobarbital would be safe, and the patient ultimately tolerated this medication. One patient (patient 26) had an adverse reaction to phenytoin (26), and, as predicted by in vitro testing, could tolerate both phenobarbital and carbamazepine.

Another patient (patient 43) had seizures secondary to cerebral metastases of lung carcinoma. He had an adverse reac-

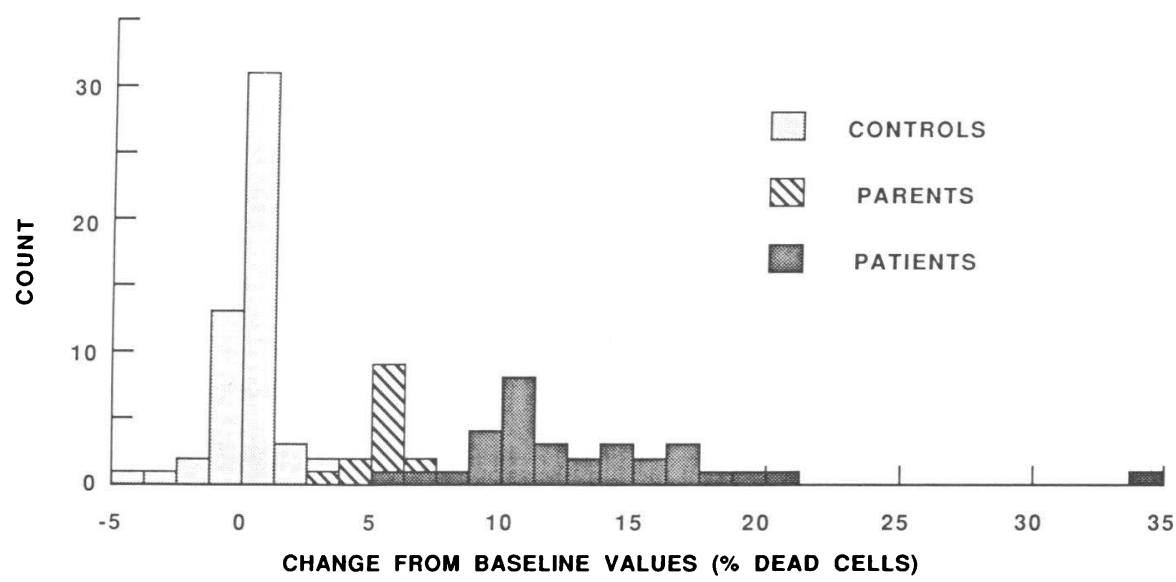

Figure 2. Histogram of in vitro results for phenytoin. The toxicity of phenytoin metabolites to cells from the parents of seven patients was intermediate to that of controls and patients. 


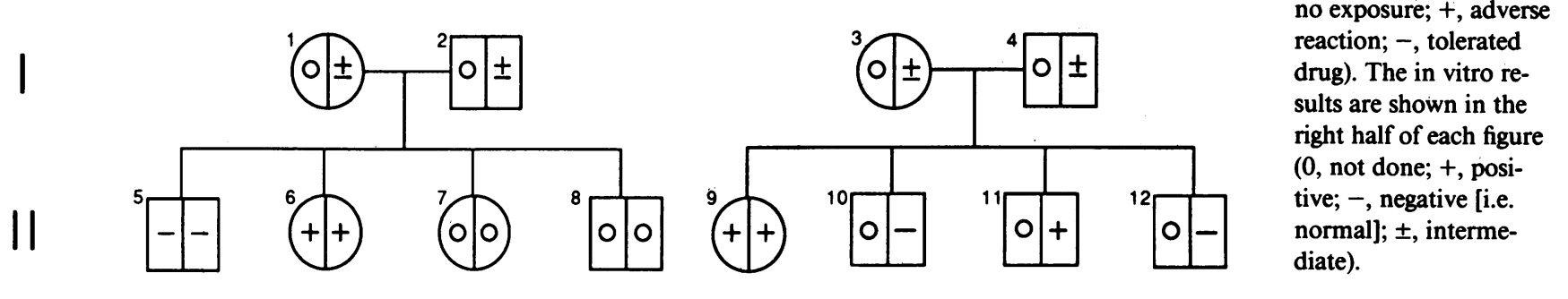

Figure 3. Outcomes of exposure to phenytoin in vivo and in vitro in two families. The results of in vivo exposure are shown in the left half of each figure $(0$, no exposure; + , adverse reaction; -, tolerated drug). The in vitro re0 , not done; + , posidiate).

tion to phenytoin, and in vitro testing suggested he was sensitive to the other anticonvulsants. Despite in vitro findings, the patient was felt to require anticonvulsant therapy, and he unfortunately had similar adverse reactions to phenobarbital and carbamazepine sequentially.

\section{Discussion}

The aromatic anticonvulsants (phenytoin, phenobarbital, and carbamazepine) are metabolized in part by cytochrome P-450 to reactive aromatic epoxide intermediates (arene oxides) $(22$, 23). The stable 10,11-epoxide of carbamazepine (not an arene oxide) was studied and found to be nontoxic to lymphocytes in vitro (Spielberg, S. P., unpublished data). Arene oxides may be responsible for cellular toxicity and the initiation of immunologic reactions. Epoxide hydrolases are cellular enzymes critical for the detoxification of arene oxides. A defect in these enzymes could lead to an accumulation of reactive metabolites though it has not been demonstrated which form of the enzyme is responsible.

Table III. Clinical (In Vivo)-In Vitro Correlation

\begin{tabular}{|c|c|c|c|c|c|c|c|c|c|c|c|c|c|c|c|c|c|c|c|c|}
\hline & Patient & 1 & 2 & 3 & 4 & 5 & 6 & 7 & 8 & 9 & 10 & 11 & 12 & 13 & 14 & 15 & 16 & 17 & 18 & 19 \\
\hline \multirow[t]{3}{*}{ Clinical } & DPH* & $1^{\ddagger}$ & 1 & 1 & - & 1 & 1 & - & 1 & - & 1 & 1 & - & 1 & 1 & 1 & - & 1 & 1 & 1 \\
\hline & PB & - & 1 & 1 & 1 & 1 & 1 & 1 & - & - & - & 1 & 1 & - & - & - & - & - & 0 & - \\
\hline & CARB & - & 1 & 1 & - & - & - & 1 & - & 1 & 1 & 1 & 1 & - & - & - & 1 & 0 & 1 & - \\
\hline \multirow[t]{4}{*}{ In vitro } & DPH & $1^{\S}$ & 1 & 1 & 1 & 1 & 1 & 1 & 1 & 1 & 1 & 1 & 1 & 1 & 1 & 1 & 1 & 1 & 1 & 1 \\
\hline & PB & 1 & 1 & 0 & 1 & 1 & 1 & 1 & 1 & 1 & 1 & 1 & 1 & 1 & 1 & - & 1 & 0 & 0 & 1 \\
\hline & CARB & 1 & 1 & 1 & 1 & 1 & 1 & 1 & 1 & 1 & 1 & 1 & 1 & 0 & 1 & 1 & 1 & 0 & 1 & 0 \\
\hline & Patient & 20 & 21 & 22 & 23 & 24 & 25 & 26 & 27 & 28 & 29 & 30 & 31 & 32 & 33 & 34 & 35 & 36 & 37 & 38 \\
\hline \multirow[t]{3}{*}{ Clinical } & DPH & - & - & 0 & 1 & 1 & 1 & 1 & 1 & 1 & 1 & - & - & - & - & 1 & - & 1 & 1 & - \\
\hline & PB & - & - & 0 & 1 & - & - & 0 & - & 1 & 1 & - & 0 & 1 & - & - & 1 & 1 & 1 & 1 \\
\hline & CARB & 1 & 1 & 1 & 1 & - & - & 0 & - & 1 & - & 1 & 1 & - & 1 & - & - & - & - & - \\
\hline \multirow[t]{4}{*}{ In vitro } & DPH & 1 & 1 & 0 & 1 & 1 & 1 & 1 & 1 & 1 & 1 & 1 & 1 & 1 & 1 & 1 & 1 & 1 & 1 & 1 \\
\hline & PB & 0 & 0 & 0 & 1 & 1 & - & 0 & - & 1 & 1 & 1 & 1 & 1 & 1 & 1 & 1 & 1 & 1 & 1 \\
\hline & CARB & 1 & 1 & 1 & 1 & 1 & - & 0 & - & 1 & 1 & 1 & 1 & 1 & 1 & 1 & 1 & 1 & 1 & 1 \\
\hline & Patient & 39 & 40 & 41 & 42 & 43 & 44 & 45 & 46 & 47 & 48 & 49 & 50 & 51 & 52 & 53 & " & 1 & & \\
\hline \multirow[t]{3}{*}{ Clinical } & DPH & - & 1 & 1 & - & 1 & 1 & - & 1 & 1 & 1 & 1 & - & 1 & - & 1 & 34 & 35 & & \\
\hline & PB & - & 1 & - & - & 1 & - & 1 & - & 0 & 1 & - & 1 & - & 1 & - & 22 & 27 & & \\
\hline & CARB & 1 & - & - & 1 & 1 & 1 & - & 1 & 1 & 1 & - & - & - & - & 1 & 25 & 27 & & \\
\hline \multirow[t]{3}{*}{ In vitro } & DPH & 0 & 1 & 1 & 1 & 1 & 1 & 1 & 1 & 1 & 1 & 1 & 1 & 1 & 1 & 1 & 51 & 53 & & \\
\hline & PB & 0 & 1 & 1 & 1 & 1 & 1 & 1 & 1 & 1 & 1 & 1 & 1 & 1 & 1 & 1 & 42 & 50 & & \\
\hline & CARB & 1 & 1 & 1 & 1 & 1 & 1 & 1 & 1 & 1 & 1 & 1 & 1 & 1 & 1 & 1 & 47 & 51 & & \\
\hline
\end{tabular}

* DPH, phenytoin; PB, phenobarbital; CARB, carbamazepine. $¥ 1$, patient received the drug and had a hypersensitivity reaction; 0 , patient received the drug and had no adverse reactions (i.e., tolerated therapy); - , patient never received the drug. $\$ 1$, positive results of in vitro lymphocyte test; 0 , negative results of in vitro lymphocyte test; - , in vitro lymphocyte test not done. "Clinical, total number of patients who had a drug reaction; In vitro, total positive test results. 'Clinical, total number of patients who received therapy; In vitro, total number of patients tested. 
An in vitro model has been developed to recreate the in vivo generation of oxidative metabolites and their subsequent detoxification. The lymphocyte is an ideal target organ that can represent the patient's ability to detoxify reactive intermediates.

In vitro testing demonstrated three distinct populations: $(a)$ normal volunteers and patients with epilepsy who tolerated phenytoin therapy, $(b)$ patients who had sustained hypersensitivity reactions to anticonvulsants, and $(c)$ the parents of patients sensitive to their drug therapy. An analysis of several families suggests that there is a clear defect in detoxification capabilities in cells from patients and that the parents' lymphocytes show an intermediate defect. Cells from the siblings of patients have been found to be either normal or equally as sensitive as patients' cells. The meaning of intermediate results or an attenuated response is not clear. None of the parents studied had been clinically exposed to anticonvulsants. However, in a study done to look at the effects of phenytoin in utero, eight mothers who were on chronic phenytoin therapy had "intermediate" values by in vitro lymphocyte toxicity testing but did not have evidence of an adverse reaction. Their offspring, who also had an "intermediate" defect, had an increased risk for major congenital malformations (27). It is possible that some patients with intermediate values do have adverse reactions when exposed to anticonvulsants but none has been studied. An autosomal codominant inheritance pattern of reactivity to anticonvulsants suggests that the patients' siblings' risk of having a reaction may be as high as one in four.

In 53 patients there were 89 exposures to anticonvulsants and 81 adverse reactions (35/36 exposed to phenytoin, $22 / 27$ to phenobarbital, and $25 / 27$ to carbamazepine). No drug appeared to be "more toxic" than another. Similarily, the clinical features of adverse reactions were similar for each drug.

The clinical syndrome of anticonvulsant hypersensitivity is characterized by the onset of fever $\sim 3 \mathrm{wk}$ after therapy has begun. 1 or $2 \mathrm{~d}$ later skin rash and lymphadenopathy develop. Still later, internal target organ disease becomes clinically apparent, with hepatitis, nephritis, and hematologic abnormalities most common. There is variability in both the target organs involved in each patient and the severity of involvement. Skin rashes were varied, and when present included mild morbilliform eruptions, persistent red and edematous plaques, erythema multiforme, or toxic epidermal necrolysis. Liver involvement ranged from mild elevations in transaminases to fulminant hepatic necrosis.

The clinical syndrome may fluctuate even in the presence of the offending drug. For example, the clearing of a suspected drug eruption during the continuation of an anticonvulsant may not mean that the drug was not responsible or that the patient is improving. Also, discontinuation of the drug (dechallenge) at an early stage may not be associated with clearing of a hypersensitivity reaction. Rather, patients may continue to deteriorate weeks after the drug has been stopped, and this should not detract from the diagnosis of an adverse reaction.

The variety of target organs involved suggests that the pathogenesis of reactions may be initiated locally rather than systematically. The organs involved (e.g., skin [epidermis], liver, kidney [tubular cells], lung [Clara cells]) are known to possess cytochromes P-450 and epoxide hydrolases (29). Organ specificity might be due to differences in expression of enzymes responsible for the toxification of drugs or detoxifi- cation of metabolites, alternate pathways of metabolism, or immunologic reactivity.

Cross-reactivity among the anticonvulsants was demonstrated both in vivo and in vitro. A structural alteration in an epoxide hydrolase could be responsible for these observations. Heterogeneous enzymatic defects may explain the subsets of patients who are intolerant to all, or to only one or two, of the anticonvulsants. Similar pharmacogenetic variation in substrate specificity has been demonstrated for other drug metabolizing enzymes, such as $N$-acetyl transferase and debrisoquine P-450 (30). Patients who have sustained hypersensitivity reactions to anticonvulsants are at high risk of reacting to the other usual drugs of choice. In light of this, the benefits of further therapy must be considered very carefully. Among our patients who needed anticonvulsants, all were able to tolerate valproic acid, but this was not always appropriate for seizure control.

We have previously shown that people sensitive to sulfonamides may have a defect in detoxification by glutathione transferases (31). The cells from these patients have apparently normal expoxide hydrolases, and cross-reactivity between the anticonvulsants and sulfonamides has not been seen.

The type of reaction seen in this study has historically been called a "hypersensitivity syndrome." This terminology is unfortunately very nonspecific and might be appropriate for a wide variety of unpredictable or immunologically mediated, drug-induced diseases. Park and his colleagues discuss the complexity of the pathophysiology of drug hypersensitivity in their review (24). The syndrome described in this paper might best be recognized as a reaction pattern with varying manifestations in response to a variety of drugs. The same pattern is not well described for nondrug etiologies and no disease-related descriptive term exists. Better nomenclature should unfold as the pathogenesis of all hypersensitivity reactions is uncovered. In the meantime we will continue to refer to the delayed, febrile, multiorgan toxicity seen here as the "anticonvulsant hypersensitivity syndrome."

The in vitro lymphocyte toxicity assay provides a model for the investigation of some hypersensitivity reactions. Our work suggests that it may aid in diagnosis and in the prediction of adverse reactions. When a simpler assay can become widely available, it would provide a rational, cost-effective approach to screening patients who are to receive anticonvulsants. For patients who are sensitive to all three aromatic anticonvulsants, an in vitro test can be used to aid the development of potentially safer new compounds. Further work needs to be done to determine the enzyme defect responsible for the limited detoxification capacity. Analogues of the major anticonvulsants could be developed for these patients and safety assessed in vitro, before in vivo challenge (i.e., therapy).

\section{Acknowledgments}

We are indebted to Dr. Michael J. Rieder and Dr. Daniel A. Goldstein for helping with the clinical care of these patients, and to the numerous physicians who kindly referred patients for consultation.

This work was supported by grant MT-7489 from the Medical Research Council of Canada. Dr. Shear is a Career Scientist of the Ontario Ministry of Health. The results and conclusions are those of the authors, and no official endorsement by the Ministry is intended or should be inferred. 


\section{References}

1. Merritt, H. H., and T. J. Putnam. 1938. Sodium diphenylhydantoinate in the treatment of convulsive disorders. JAMA (J. Am. Med. Assoc.). 111:1068-1073.

2. Merritt, H. H., and T. J. Putnam. 1939. Sodium diphenylhydantoinate in treatment of convulsive disorders: toxic symptoms and their prevention. Arch. Neurol. Psychiatry. 42:1053-1058.

3. Wilson, J. T., B. Höjer, G. Tomson, A. Rane, and F. Sjöqvist. 1978. High incidence of concentration-dependent skin reactions in children. Br. Med. J. 1:1583-1586.

4. Chaiken, R. H., B. I. Goldberg, and J. P. Segal. 1950. Dilantin sensitivity: report of a case of hepatitis with jaundice, pyrexia and exfoliative dermatitis. $N$. Engl. J. Med. 242:897-898.

5. Haruda, F. 1979. Phenytoin hypersensitivity: 38 cases. Neurology. $29: 1480-1485$. 541.

6. Tomsick, R. S. 1983. The phenytoin syndrome. Cutis. 32:535-

7. Michael, J. R., and M. L. Rudin. 1981. Acute pulmonary disease caused by phenytoin. Ann. Intern. Med. 95:452-454.

8. Hyman, L. R., M. Ballow, and M. R. Knieser. 1978. Diphenylhydantoin interstitial nephritis: roles of cellular and humoral immunologic injury. J. Pediatr. 92:915-920.

9. Smith, D. A., and W. H. G. Burgdorf. 1984. Universal cutaneous depigmentation following phenytoin-induced toxic epidermal necrolysis. J. Am. Acad. Dermatol. 10:106-109.

10. Sherertz, E. F., B. V. Jegasothy, and G. S. Lazarus. 1985. Phenytoin hypersensitivity reaction presenting with toxic epidermal nercolysis and severe hepatitis: report of a patient treated with corticosteriod "pulse therapy." J. Am. Acad. Dermatol. 12:178-181.

11. Gerson, W. T., D. G. Fine, S. P. Spielberg, and L. L. Sensenbremner. 1983. Anticonvulsant induced aplastic anemia: increased susceptibility to toxic drug metabolites in vitro. Blood. 61:889-893.

12. McGeachy, T. E., and W. E. Bloomer. 1953. The phenobarbital sensitivity syndrome. Am. J. Med. 14:600-604.

13. Jeavons, P. M. 1983. Hepatotoxicity of antiepileptic drugs. In Antiepileptic Therapy: Chronic Toxicity of Antiepileptic Drugs. J. Oxley, editor. Raven Press, New York. 1-45.

14. Crill, W. E. 1973. Carbamazepine. Ann. Intern. Med. 79:844847.

15. Breathnach, S. M., D. H. McGibbon, F. A. Ive, and M. M. Black. 1982. Carbamazepine ("Tegretol") and toxic epidermal necrolysis: report of three cases with histopathologic observations. Clin. Exp. Dermatol. 7:585-591.

16. Pisciotta, A. V. 1975. Hematologic toxicity of carbamazepine. In Advances in Neurology. J. K. Penry and D. D. Daly, editors. Raven Press, New York. 355-368.
17. Shuttleworth, D., R. A. C. Graham-Brown, A. J. Williams, A. C. Campbell, and H. Sewell. 1984. Pseudo-lymphoma associated with carbamazepine. Clin. Exp. Dermatol. 9:421-423.

18. De Swerte, L. F., J. L. Ceuppens, D. Teuwen, L. Wijndaele, P. Caesar, and M. Casteels-Van Daele. 1984. Acute interstitial pneumonitis and carbamazepine therapy. Acta Paediatr. Scand. 73:285288.

19. Saltzstein, S. L., and L. V. Ackerman. 1959. Lymphadenopathy induced by anticonvulsant drugs and mimicking clinically and pathologically malignant lymphomas. Cancer (Phila.). 12:164-182.

20. Pollack, M. A., P. G. Burk, and G. Nathanson. 1979. Mucocutaneous eruptions due to antiepileptic drug therapy in children. Ann. Neurol. 5:262-267.

21. Kahn, H. D., G. B. Faguet, J. F. Agee, and H. M. Middleton. 1984. Drug-induced liver injury: in vitro demonstration of hypersensitivity to both phenytoin and phenobarbital. Arch. Intern. Med. 144:1677-1679.

22. Lertratanangkoon, K., and M. G. Horning. 1982. Metabolism of carbamazepine. Drug Metab. Dispos. 10:1-10.

23. Pantarotto, C., M. Arboix, P. Sezzano, and R. Abbruzzi. 1982. Studies on 5,5-diphenylhydantoin irreversible binding to rat liver microsomal proteins. Biochem. Pharmacol. 31:1501-1507.

24. Park, B. K., J. W. Coleman, and N. R. Kitteringham. 1987. Drug disposition and drug hypersensitivity. Biochem. Pharmacol. 36:581-590.

25. Spielberg, S. P., G. B. Gordon, D. A. Blake, E. D. Mellits, and D. S. Bross. 1981. Anticonvulsant toxicity in vitro: possible role of arene oxides. J. Pharmacol. Exp. Ther. 217:386-389.

26. Spielberg, S. P., G. B. Gordon, D. A. Blake, D. A. Goldstein, and H. F. Herlong. 1981. Predisposition to phenytoin hepatotoxicity assessed in vitro. N. Engl. J. Med. 305:722-727.

27. Strickler, S. M., L. V. Dansky, M. A. Miller, M.-H. Seni, E. Andermann, and S. P. Spielberg. 1985. Genetic predisposition to phenytoin-induced birth defects. Lancet. ii:746-749.

28. Spielberg, S. P., and G. B. Gordon. 1981. Nitrofurantoin cytotoxicity: in vitro assessment of risk based on glutathione metabolism. J. Clin. Invest. 67:37-41.

29. Bend, J. R., and C. J. Serabijt-Singh. 1984. Xenobiotic metabolism by extrahepatic tissues: relationship to target organ and cell toxicity. In Drug Metabolism and Drug Toxicity. J. R. Mitchell and M. G. Horning, editors. Raven Press, New York. 99-136.

30. Kalow, W. 1987. Genetic variation in the human hepatic cytochrome P-450 system. Eur. J. Clin. Pharmacol. 31:633-641.

31. Shear, N. H., S. P. Spielberg, D. M. Grant, B. K. Tang, and W. Kalow. 1986. Differences in metabolism of sulfonamides predisposing to idiosyncratic toxicity. Ann. Intern. Med. 105:179-84. 